\title{
Eupafolin inhibits breast cancer cell proliferation and induces apoptosis by inhibiting the PI3K/Akt/mTOR pathway
}

\author{
JIAHUI WEI $^{1 *}$, XUEFENG ZHANG ${ }^{2 *}$, HUIHAO PAN ${ }^{1}$, SONG HE $^{1}$, \\ BAO YUAN ${ }^{1}$, QING LIU ${ }^{3}$, JIABAO ZHANG ${ }^{1}$ and YU DING $^{1}$

\begin{abstract}
${ }^{1}$ Department of Laboratory Animals, College of Animal Sciences, Jilin University, Changchun, Jilin 130062; ${ }^{2}$ College of Animal Science and Technology, Jilin Agricultural University, Changchun, Jilin 130118 ; ${ }^{3}$ The Second Clinical School of Medicine, Guangdong Provincial Hospital of Chinese Medicine, Guangdong Provincial Hospital of Chinese Medicine-Zhuhai Hospital, Guangzhou University of Chinese Medicine, Guangzhou, Guangdong 510120, P.R. China
\end{abstract}

Received September 23, 2020; Accepted December 29, 2020

DOI: $10.3892 / \mathrm{ol} .2021 .12593$

\begin{abstract}
Eupafolin is a flavonoid extracted from common sage. Previous studies have reported that Eupafolin has antioxidant, anti-inflammatory, and anti-tumor effects. However, its role in breast cancer remains unclear. The present study investigated the effects and underlying mechanism of action of Eupafolin using breast cancer cell lines. The effects of Eupafolin on breast cancer cell proliferation, migration, apoptosis and the cell cycle were determined. Cell viability and Transwell assays, reverse transcription-quantitative PCR, flow cytometry and western blot analysis were used in this study. The data showed that the proliferation, migration and invasion ability of EO771 cells treated with Eupafolin was significantly decreased, and the apoptosis rate was increased compared with that of the control. The protein levels of Bax and cleaved caspase 3 increased, whereas that of Bcl-2 decreased. In addition, Eupafolin treatment also caused the proliferation of breast cancer cells to be arrested at the $G_{0} / G_{1}$ phase. Furthermore, results from western blotting indicated that Eupafolin treatment decreased the protein levels of p-PI3K, p-Akt and p-mTOR. Taken together, the present findings demonstrate that Eupafolin has a significant inhibitory effect on the proliferation of EO771 cells, inhibits cell migration and invasion, and promotes cell apoptosis, thereby causing $G_{0} / G_{1}$ phase arrest, at least partially through the PI3K/Akt/mTOR signaling pathway. Therefore, the findings provide novel insights regarding the use of Eupafolin for the treatment of breast cancer.
\end{abstract}

Correspondence to: Dr Jiabao Zhang or Dr Yu Ding, Department of Laboratory Animals, College of Animal Sciences, Jilin University, 5333 Xi'an Road, Changchun, Jilin 130062, P.R. China

E-mail: zjb515@163.com

E-mail: dingyu03@163.com

${ }^{*}$ Contributed equally

Key words: apoptosis, breast cancer, Eupafolin, PI3K/Akt/mTOR, proliferation

\section{Introduction}

Breast cancer is one of the most dangerous invasive cancer in women that has a global prevalence (1). Although the overall mortality rate of patients with breast cancer has decreased, it is continuing to emerge as a major health issue in women worldwide. At present, surgical treatment is the best option. Chemotherapy and radiotherapy are used to inhibit the growth and spread of tumors, and after many years of medical advancement and improvement, both chemotherapy and radiotherapy have been shown to prolong the lives of patients (2). The proliferation and recurrence rates of breast cancer cells are very high, and some patients develop drug resistance, which may cause side effects. Therefore, identifying non-toxic and efficacious natural compounds for the treatment of breast cancer is of utmost importance.

Traditional Chinese medicine has been widely used in China. Due to its non-toxic effects and efficacy, it is often used in combination with other medicines. With the continuous progress in modern medicine, preventing and treating the recurrence and metastasis of breast cancer using genetic technology and molecular biology methods will become a trend in future breast cancer research. Eupafolin is a flavonoid, which has anti-inflammatory, anti-viral, anti-angiogenesis and anti-tumor activities (3). Angiogenesis is closely associated with tumor development and metastasis, and Eupafolin can inhibit the activation of VEGFR2 and its associated signaling pathways. The molecular mechanism of the anti-cancer effect of Eupafolin may be associated with the activation of caspase-3 (4), the downregulation of vascular endothelial growth factor (VEGF) (5), and inhibition of the Akt signaling pathway (6). However, the underlying mechanism of its anticancer effect in breast cancer remains unclear. Therefore, understanding the effect of Eupafolin on breast cancer and identifying the mechanism of action will help in the management of breast cancer.

Proteins involved in the PI3K/Akt/mTOR pathway are abnormally expressed in several tumors, thereby leading to the progression of breast cancer, gastric cancer, nasal cancer and pancreatic cancer, among others. This pathway is closely associated with tumor proliferation, autophagy 
and migration $(7,8)$. Several studies show that targeting this pathway using drugs or drug combinations is effective in the treatment of tumors (9). Therefore, research on drugs targeting the PI3K/Akt/mTOR pathway may have great significance in the management of breast cancer.

Therefore, the aim of the present study was to investigate whether Eupafolin could inhibit the proliferation and apoptosis of breast cancer cells (EO771 cell line), and to identify its possible underlying mechanism of action. Experimental results showed that Eupafolin significantly inhibited the proliferation of EO771 cells by modulating the PI3K/Akt/mTOR pathway, causing $\mathrm{G}_{0} / \mathrm{G}_{1}$ phase arrest, and promoting apoptosis.

\section{Materials and methods}

Cell culture and processing. The mouse breast cancer cell line, EO771, selected for the present study was obtained from Binsui Biotechnology Co., Ltd. EO771 cells were cultured in Dulbecco's Modified Eagle's Medium (DMEM; Gibco; Thermo Fisher Scientific Inc.), with $10 \%$ fetal bovine serum (Gibco; Thermo Fisher Scientific, Inc.), and maintained in an atmosphere of $5 \% \mathrm{CO}_{2}$ in an incubator at $37^{\circ} \mathrm{C}$. Eupafolin (purity $\geq 99 \%$ ) was purchased from Yuanye Biotechnology. In the present study, Eupafolin was dissolved in dimethyl sulfoxide (DMSO; Beijing Solarbio Science \& Technology Co., Ltd.) at different concentrations (0, 25, 50 and $100 \mu \mathrm{M})$. Subsequently, cells were treated with Eupafolin for different periods.

Cell viability test. Cell viability was determined using Cell Counting kit-8 (CCK-8; MedChemExpress). In brief, 5,000 EO771 cells were seeded into 96-well plates, treated with $0,25,50$ and $100 \mu \mathrm{M}$ Eupafolin, and incubated at $37^{\circ} \mathrm{C}$ for 24,36 and $48 \mathrm{~h}$. Then, $10 \mu \mathrm{l} \mathrm{CCK}-8$ solution was added to each well and the cells were incubated for $1.5 \mathrm{~h}$. A microplate reader was used to measure the absorbance at $450 \mathrm{~nm}$ (TECAN).

Determination of apoptosis and cell cycle. Apoptosis was measured according to the instructions provided with the Annexin-V/Fluorescein isothiocyanate (FITC) apoptosis detection kit (Ebisson). After drug treatment for $24 \mathrm{~h}$, cells were digested with trypsin without EDTA, collected, centrifuged at $500 \times \mathrm{g}$ for $5 \mathrm{~min}$ at $37^{\circ} \mathrm{C}$, resuspended in pre-cooled PBS, centrifuged at $500 \mathrm{x} \mathrm{g}$ for $5 \mathrm{~min}$ to discard the supernatant, resuspended in $300 \mu 11 \mathrm{X}$ binding buffer; $5 \mu \mathrm{l}$ sample was mixed with FITC-Annexin V. Then, $5 \mu \mathrm{l}$ propidium iodide (PI) staining solution was added to $200 \mu \mathrm{l}$ $1 \mathrm{X}$ binding buffer $5 \mathrm{~min}$ prior to detection. Flow cytometry was performed using FACS (Thermo Fisher Scientific, Inc.). For cell cycle analysis, the cells were harvested and fixed in $70 \%$ ethanol at $4^{\circ} \mathrm{C}$ overnight. Next, cells were resuspended in $500 \mu \mathrm{l} 1 \mathrm{X}$ PI solution (Baihao) for $30 \mathrm{~min}$ at $37^{\circ} \mathrm{C}$. Flow cytometry analysis was performed using FACS (Thermo Fisher Scientific, Inc.). The collected data were analyzed using ModFit LT (version 2.0; Verity Software House, Inc.) to determine cell cycle distribution.

Cell scratch test. A total of 10,000 EO771 cells was seeded into 6-well culture plates in DMEM containing 10\% FBS, and placed in an incubator until the cell density was $90 \%$ or higher.
Using a sterile $100-\mu 1$ plastic pipette, a wound was created; the cell debris was removed by washing with PBS and images were captured using an inverted light microscope (Olympus Corporation) with a digital camera (magnification, x80) at $0 \mathrm{~h}$. A total of $3 \mathrm{ml}$ of FBS-free medium was added per well; then, 25, 50 and $100 \mu \mathrm{M}$ Eupafolin was added for $24 \mathrm{~h}$. Cells were washed with PBS and images were obtained using microscopy. Healing areas were analyzed using ImageJ software (version 1.51; National Institutes of Health). The experiment was performed at least in triplicate.

Cell migration and invasion experiments. A Transwell (Corning, Inc.) assay was performed to determine cell migration and invasion abilities. After Eupafolin treatment, cells to be tested in the logarithmic growth phase were digested, resuspended in serum-free medium, and

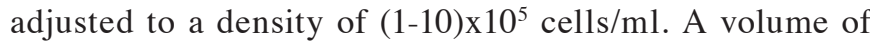
$500 \mu 1$ DMEM containing 10\% FBS was added to the lower chamber of each transwell; tweezers were used to place the cells in a new 24-well plate. A volume of $100 \mu \mathrm{l}$ of the cell suspension was added to the upper chamber, and the transwells were placed in an incubator for $24 \mathrm{~h}$. Next, the cells were removed and the medium was aspirated. In a new 24 -well plate, $600 \mu 14 \%$ paraformaldehyde was added. The transwell was placed at room temperature and cells were fixed for 20-30 min. The fixative solution was removed and cells were stained at $37^{\circ} \mathrm{C}$ using $0.2 \%$ crystal violet for $10 \mathrm{~min}$. Then, the cells were washed three times with PBS to remove the unbound crystal violet. The upper surface of the chamber was gently wiped with a cotton swab. Excess crystal violet was removed prior to microscopy. After drying, five fields were randomly selected and the cells were observed and counted under an inverted light microscope with a digital camera (magnification, $x 80$ ). The cell invasion test procedure that was used was similar to that of the cell migration assay, except that the upper chamber was covered with BD Matrigel $^{\mathrm{TM}}$ Matrix (Corning, Inc.).

Reverse transcription-quantitative PCR (RT-qPCR). According to the instructions provided with the kit, TRIzol reagent (Ruan) was used to extract total RNA, and cDNA was generated using the FastKing RT kit (Tiangen Biotech Co., Ltd) with $2 \mu \mathrm{g}$ RNA, according to the manufacturer's instructions. The primers used were obtained from NCBI. A real-time fluorescent quantitative PCR detection system (Eppendorf) was used to perform the RT-qPCR reactions using SYBR-Green (Tiangen Biotech Co., Ltd.) and a total of $20 \mu \mathrm{l}$ reaction mixture. The $2^{-\Delta \Delta \mathrm{Cq}}$ method (10) was used to analyze gene expression levels. Primer sequences were as follows: Matrix metalloprotease (MMP)2 forward, 5'-CTGCAGGTG GTCATAG-3' and reverse, 5'-TGGTGTGCAGCGATGAAG AT-3'; MMP9 forward, 5'-CTTCACCGGCTAAACCACCT-3' and reverse, 5'-CTTCACCGGCTAAACCACCT-3'; VEGFA forward, 5'-ATAGGAGAGATGAGCTTCC-3' and reverse, 5'-TCTGCATTCACATCTGCTGTGC-3'; $\beta$-actin forward, 5'-GTCGAGTCGCGTCCACC-3' and reverse, 5'-GTCATC CATGGCGAACTGGT-3'; cyclin D1 forward, 5'-CTGTGC TGCGAAGTGGAAACCAT-3' and reverse, 5'-TTCATGGCC AGCGGGAAGACCTC-3'; CDK4 forward, 5'-CGAGCGTAA GGCTGATGGAT-3' and reverse, 5'-CCAGGCCGCTTA 
A

Molecular formula: $\mathrm{C} 16 \mathrm{H} 12 \mathrm{O}$ Molecular weight: 316.267<smiles>CCOCCN(CC)CC</smiles>

B

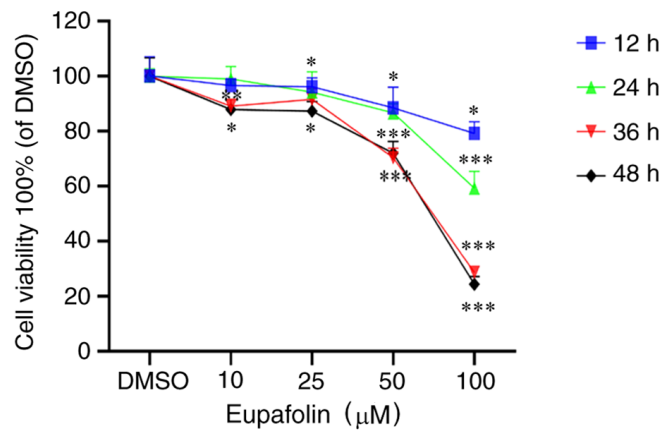

Figure 1. Eupafolin decreases the proliferation of EO771 cells. (A) Molecular structure of Eupafolin. (B) EO771 cells were exposed to various concentrations of Eupafolin $(0,25,50$ and $100 \mu \mathrm{M})$ for different durations, and the cell viability was determined using the Cell Counting Kit-8 assay. Data are shown as mean \pm standard deviation from three independent experiments. ${ }^{*} \mathrm{P}<0.05 ;{ }^{* * *} \mathrm{P}<0.001$ vs. group.

GAAACTGA-3'; and CDK6 forward, 5'-AGCCCTGCTGTG GAAGAAAA-3' and reverse, 5'-TAGACGGACCGACCTTCT CG-3'. The qPCR reaction conditions were as follows: Initial denaturation for $15 \mathrm{~min}$ at $95^{\circ} \mathrm{C}$, followed by 40 cycles of denaturation for $10 \mathrm{sec}$ at $95^{\circ} \mathrm{C}$, annealing for $30 \mathrm{sec}$ at $60^{\circ} \mathrm{C}$ and extension for $20 \mathrm{sec}$ at $72^{\circ} \mathrm{C}$.

Western blotting. After treating the cells with Eupafolin for 24 h, EO771 cells were harvested, washed twice with PBS, and lysed on ice with RIPA lysis buffer (Beijing Solarbio Science \& Technology Co., Ltd.) for $30 \mathrm{~min}$. Then, the BCA method was used to determine the protein concentration: $5 \mathrm{X}$ loading buffer was added and proteins were denatured at $95^{\circ} \mathrm{C}$ by boiling in a water bath for $10 \mathrm{~min}$. A total of $20 \mu \mathrm{g}$ protein sample was added to each well, separated using $12 \%$ SDS-PAGE at $120 \mathrm{~V}$, and then transferred to PVDF membranes (Thermo Fisher Scientific, Inc.). Skimmed milk powder (5\%) was used to block the PVDF membranes for $1 \mathrm{~h}$ at room temperature. Next, the membranes were incubated with primary antibodies directed against Bcl-2 (cat. no. 4223), Bax (cat. no. 2772), cleaved caspase 3 (cat. no. 9661), PI3K (cat. no. 4257), p-PI3K (cat. no. 17366), Akt (cat. no. 4691), p-Akt (cat. no. 4060), Mtor (cat. no. 2983), p-mTOR (cat. no. 5536) and GAPDH (cat. no. 5174) (all 1:1,000; Cell Signaling Technology, Inc.) at $4^{\circ} \mathrm{C}$ overnight. Subsequently, membranes were washed three times with PBS, then incubated with a corresponding horseradish peroxidase-conjugated secondary antibody $(1: 2,000$; cat. no. A0208; Beyotime Institute of Biotechnology) for $1.5 \mathrm{~h}$ at room temperature and washed five times with PBS. Then, protein bands were visualized using an enhanced chemiluminescence assay kit (Beyotime Institute of Biotechnology) and photographed using an imaging system (Tanon). Finally, data were analyzed using ImageJ software (version 1.8.0; National Institutes of Health).

Statistical analysis. All experiments were repeated $\geq 3$ times. The data are presented as the mean $\pm \mathrm{SD}$ and were analyzed using SPSS (v.20.0; IBM Corp.). Differences between multiple groups were analyzed using one-way ANOVA followed by Dunnett's post hoc test. Results with $\mathrm{P}<0.05 ; \mathrm{P}<0.01$; and $\mathrm{P}<0.001$ were considered as statistically significant; 'ns' indicates $\mathrm{P}>0.05$.

\section{Results}

Eupafolin decreases the viability and proliferation of breast cancer cells. EO771 cells were treated with Eupafolin for 24, 36 and $48 \mathrm{~h}$ to study the effects of the compound (Fig. 1A) on cell proliferation. The results of the CCK-8 assay indicated that Eupafolin inhibited the viability of EO771 cells, and the inhibitory effect was proportional to the treatment time and dose (Fig. 1B).

Eupafolin inhibits the invasion and migration of breast cancer cells. The scratch test was used to determine the wound healing time of EO771 cells. Furthermore, a Transwell assay was performed to determine the ratio of invasion and migration of EO771 cells (Fig. 2A and B). Results showed that compared with the control cells, Eupafolin significantly decreased the migration and invasion of EO771 cells (Fig. 2C). MMP2, MMP9 and VEGF-A are positively associated with the migration ability of tumor cells and can be used as marker genes. Therefore, their expression following Eupafolin treatment was tested, and the results showed that Eupafolin inhibited MMP2, MMP9 and VEGF-A (Fig. 2D). Taken together, these results indicate that Eupafolin prevented further deterioration of breast cancer cells.

Eupafolin induces apoptosis of breast cancer cells. Next, whether Eupafolin could induce apoptosis in breast cancer cells was tested. Flow cytometry was used to analyze the apoptosis ratio of EO771 cells following treatment with Eupafolin. The results showed that compared with the control, $100 \mu \mathrm{M}$ Eupafolin increased the apoptosis rate of EO771 cells by 18\% (Fig. 3A). To further understand the specific mechanism of Eupafolin in causing apoptosis in EO771 cells, the expression of apoptosis-associated proteins was evaluated using western blotting. The protein levels of cleaved caspase 3 and Bax were increased, whereas Bcl-2 protein levels were decreased (Fig. 3B and C).

Eupafolin induces $G_{0} / G_{1}$ phase arrest in breast cancer cells. After staining with PI, cell cycle analysis of EO771 cells treated with various concentrations of Eupafolin was performed using flow cytometry. Eupafolin induced $\mathrm{G}_{0} / \mathrm{G}_{1}$ phase arrest in EO771 cells (Fig. $4 A$ and B). In 
A
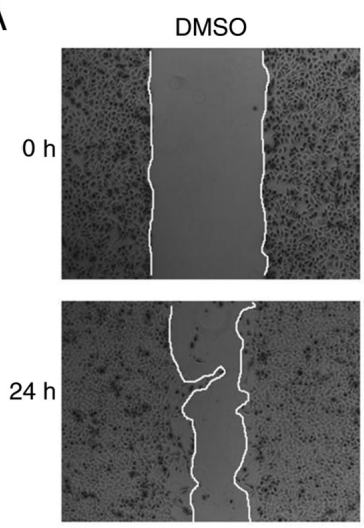

$25 \mu \mathrm{M}$
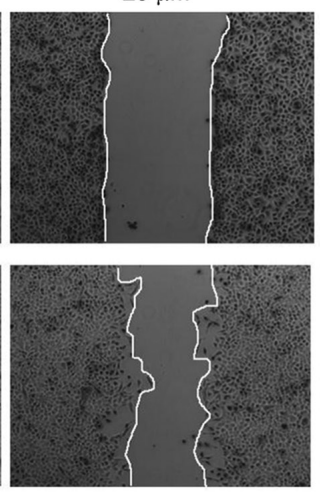

$50 \mu \mathrm{M}$
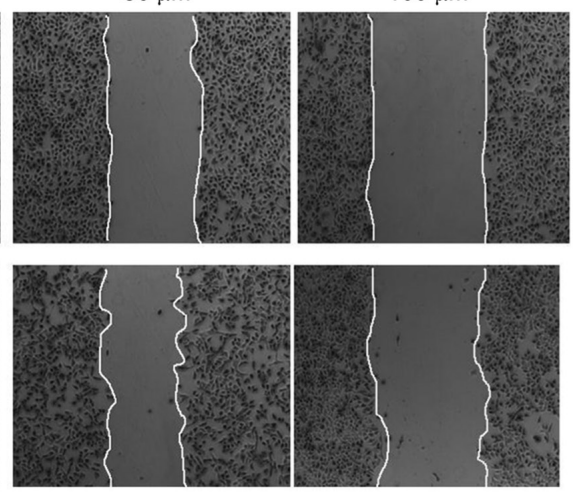

B

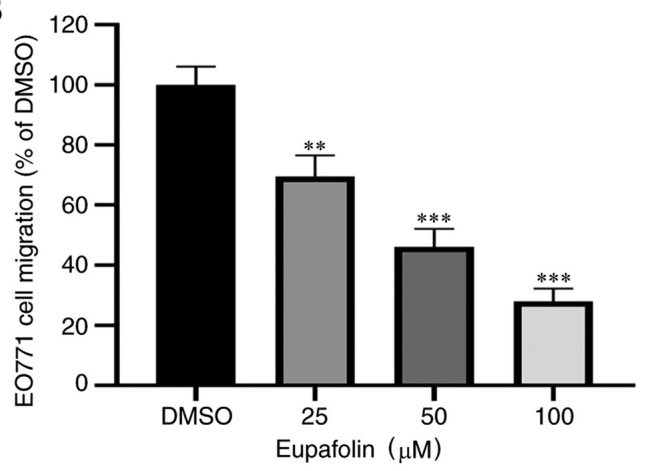

C

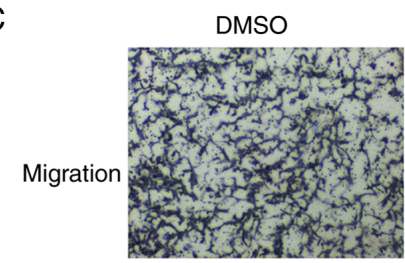

$25 \mu \mathrm{M}$

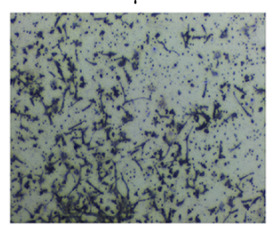

$50 \mu \mathrm{M}$
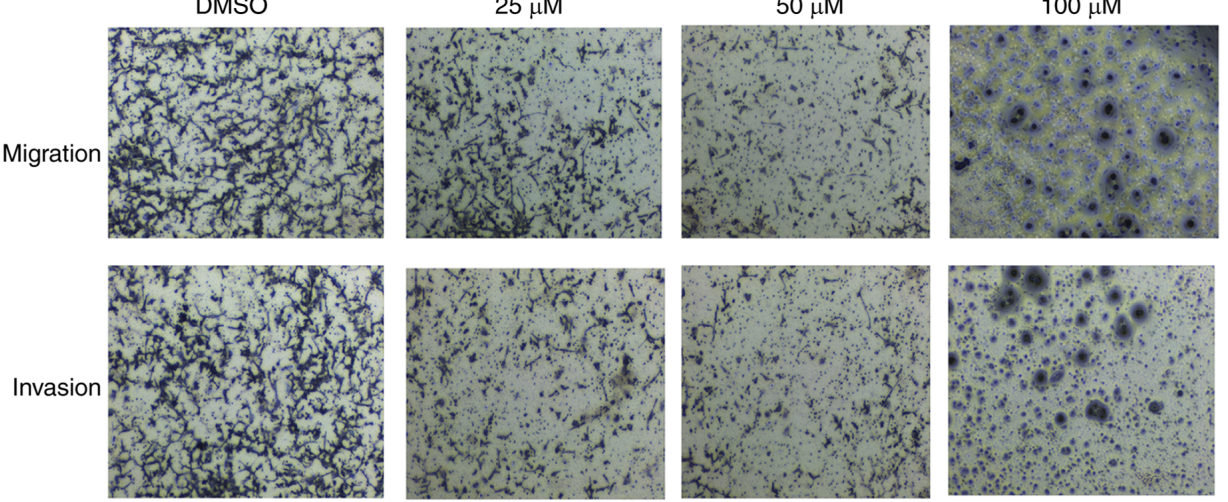

D

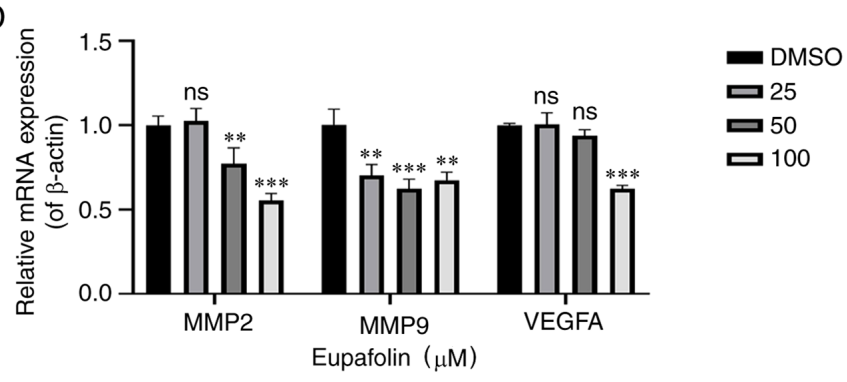

Figure 2. Eupafolin attenuates the invasion and migration of EO771 cells. After seeding EO771 cells in a 6-well plate and treating them with different concentrations of Eupafolin for $24 \mathrm{~h}$, the migration distance of the cells was measured, the proportion of invaded cells was calculated, and RNA was extracted from cells to determine the expression of associated genes using RT-qPCR. (A and B) $24 \mathrm{~h}$ after Eupafolin treatment, the healing effect of EO771 cells was determined using the scratch test. (C) The ratio of invasion and migration of EO771 cells was further determined using a transwell assay. (D) EO771 cells were harvested at $24 \mathrm{~h}$ post treatment for RT-qPCR to determine the mRNA expression of MMP2, MMP9 and VEGF-A. Data are expressed as the mean \pm SD. ns, $\mathrm{P}>0.05 ;{ }^{* *} \mathrm{P}<0.01 ;{ }^{* * * *} \mathrm{P}<0.001$. MMP, matrix metallopeptidase; RT-qPCR, reverse transcription-quantitative PCR; VEGF, vascular endothelial growth factor.

addition, RT-qPCR was used to determine the mRNA levels of cycle-associated genes. The results showed that Eupafolin inhibited the expression of cyclin D1, CDK4 and CDK6 mRNA (Fig. 4C).
Eupafolin downregulates the PI3K/Akt/mTOR pathway. The aforementioned data indicated that Eupafolin had an impact on the proliferation and apoptosis of EO771 cells. $\mathrm{PI} 3 \mathrm{~K} / \mathrm{Akt} / \mathrm{mTOR}$ was found to play an important role in the 
A
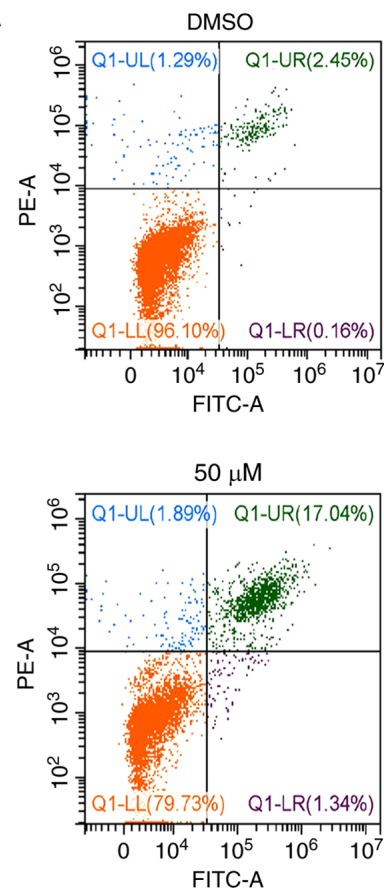
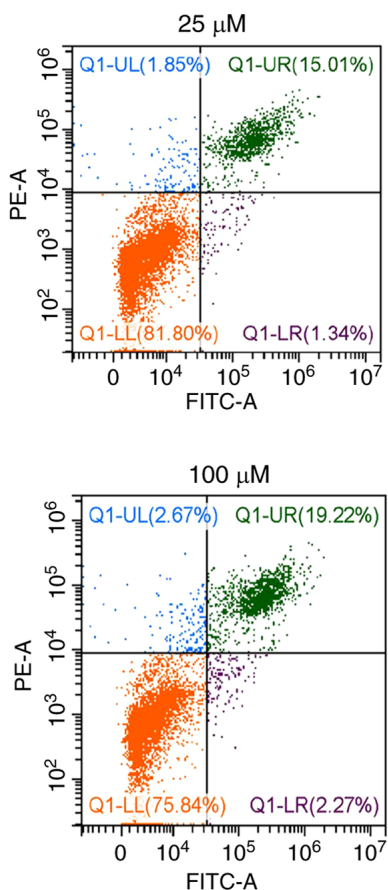

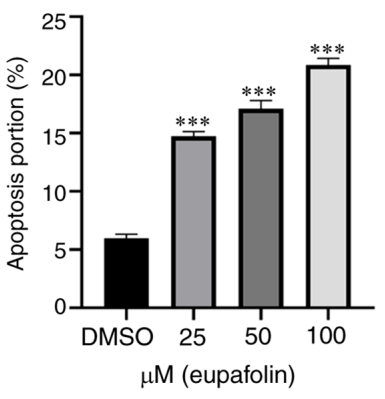

B
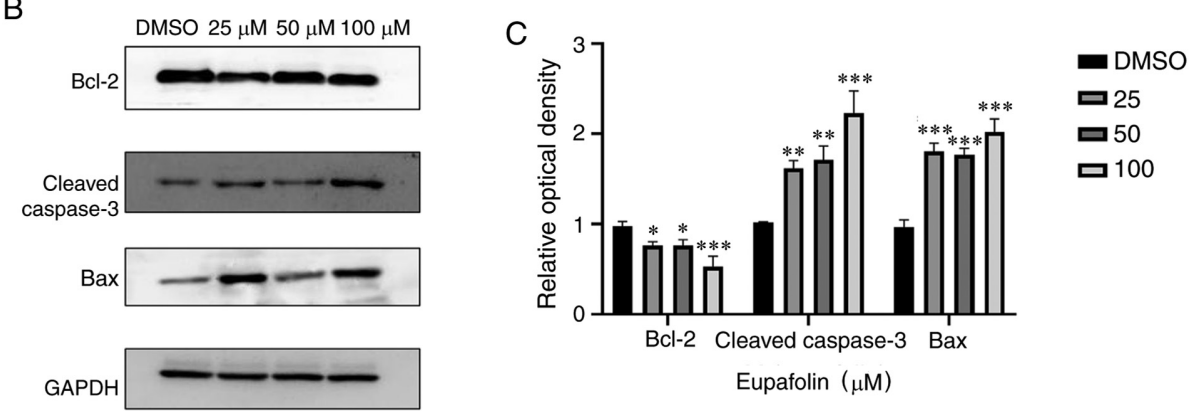

Figure 3. Eupafolin induces apoptosis in EO771 cells. (A) EO771 cells were treated with 25, 50 and $100 \mu \mathrm{M}$ Eupafolin for 24 h. Cells were then digested and the proportion of apoptosis of breast cancer cells was determined using flow cytometry. (B) After extraction and treatment, the levels of cleaved caspase 3 , Bax, and Bcl-2 proteins were determined using western blotting. (C) Western blots were analyzed using Image J software and compared with those of the control group. Data are representative of three independent experiments and expressed as the mean $\pm \mathrm{SD} . \mathrm{ns}, \mathrm{P}>0.05 ;{ }^{* * *} \mathrm{P}<0.001 ;{ }^{* *} \mathrm{P}<0.01 ;{ }^{*} \mathrm{P}<0.05$.

occurrence of many tumors. Therefore, whether Eupafolin affected EO771 cells through this pathway was tested. Results from western blotting showed that Eupafolin significantly decreased protein levels of p-PI3K, p-Akt and p-mTOR. Taken together, these results indicate that Eupafolin affected the proliferation and apoptosis of EO771 cells through the PI3K/Akt/mTOR pathway (Fig. 5A and B).

\section{Discussion}

The GLOBOCAN 2018 Global Cancer Analysis Annual Report released by the World Health Organization in 2018 indicates that annually, 2.088 million new cases of breast cancer and 627,000 deaths occur worldwide (1). China's incidence rate ranks 120 in the world (11). Although the total incidence of breast cancer in China is lower than that in other developed countries, the trend shows a gradual increase (1). Current breast cancer treatment strategies mainly focus on chemotherapy and radiotherapy; however, these treatment strategies almost inevitably have side effects, which have become the bottleneck of clinical tumor treatment (12). Since natural compounds have advantages of higher efficacy and lower toxicity, many natural compounds have been used clinically (13). Eupafolin is a natural compound extracted from plants. Previous studies have reported that Eupafolin has anti-inflammatory and anti-tumor effects (3). For example, Eupafolin can inhibit the viability of esophageal cancer cells in vivo and in vitro by targeting T-LAK, and by downregulating Mcl-1 and upregulating Mcl-1 (4). Bim enhances TRAIL-mediated apoptosis of renal cancer cells (14). In addition, Eupafolin can induce apoptosis in HeLa human cervical cancer cells by inhibiting the expression of associated apoptosis proteins (3). However, the role of Eupafolin in breast cancer and its possible underlying mechanism of action have not yet been elucidated. Therefore, the present study aimed to determine the effect of Eupafolin on breast cancer cells. Our results showed that Eupafolin treatment had a significant inhibitory effect on breast cancer cell proliferation, promoted cell apoptosis, and induced $\mathrm{G}_{0} / \mathrm{G}_{1}$ phase arrest. Therefore, the data indicated that Eupafolin could inhibit the proliferation of breast cancer cells and induce apoptosis thereof, via downregulating the $\mathrm{PI} 3 \mathrm{~K} / \mathrm{Akt} / \mathrm{mTOR}$ pathway. 
A
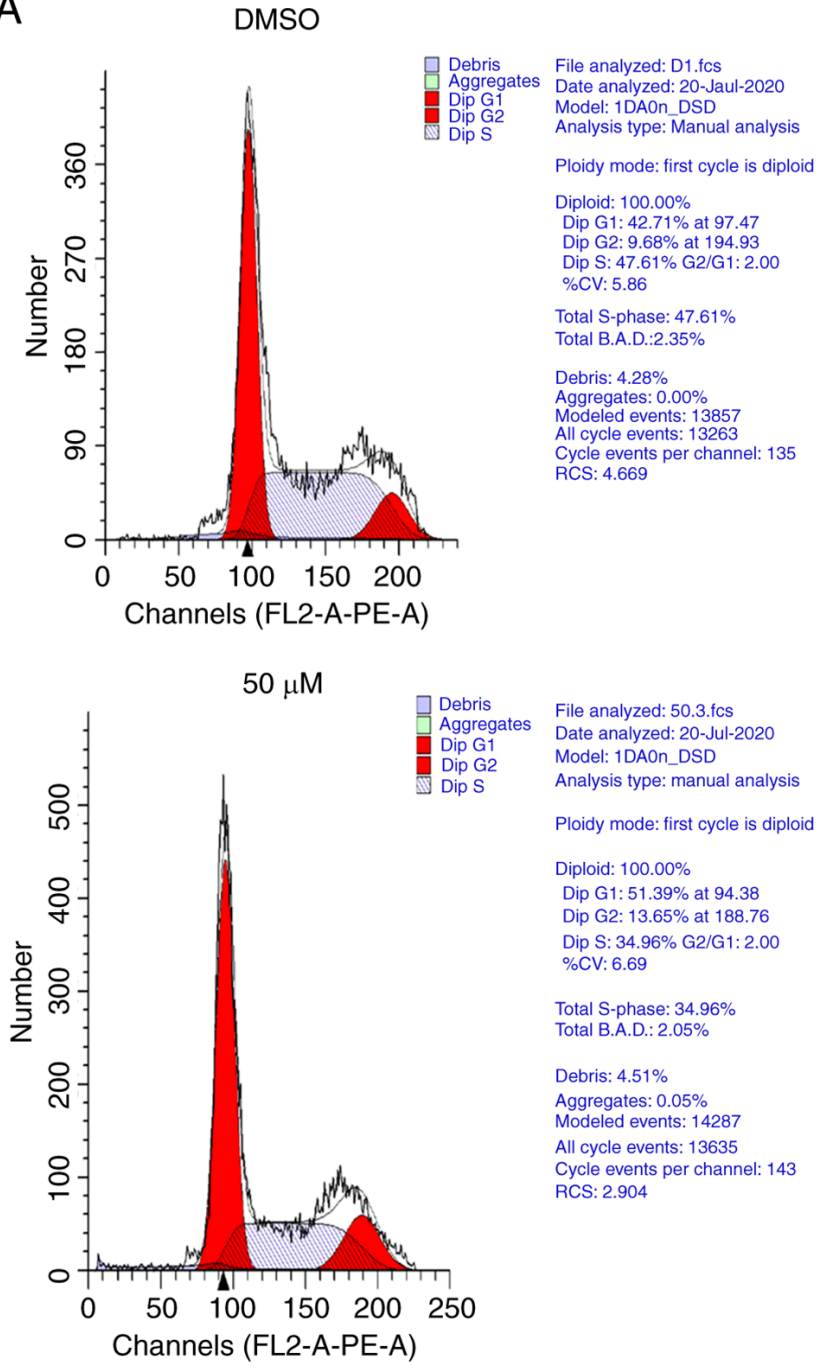

$25 \mu \mathrm{M}$

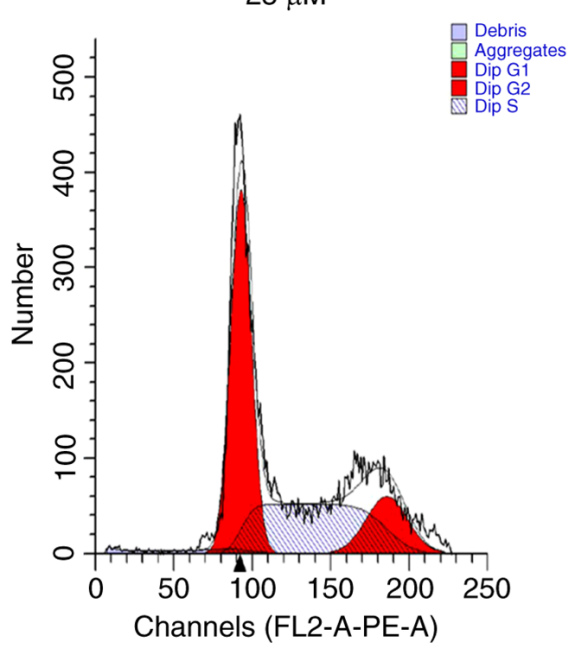

$100 \mu \mathrm{M}$
File analyzed: $25-3 . f c s$ Dale analyzed: 20-Jul-2020 Analysis type: man

manual analysis

Ploidy mode: first cycle is diploid

Diploid: $100.0 \%$

Dip G1: $48.26 \%$ at 92.80 Dip G2: $15.07 \%$ at 185.60 Dip S: $36.67 \%$ G2/G1: 2.00 \%CV: 7.14

Total S-phase: $36.67 \%$ Total B.A.D.: $1.34 \%$

Debris: $3.19 \%$

Aggregates: $0.17 \%$

Modeled events: 13638

Cycle

CS: 3.543
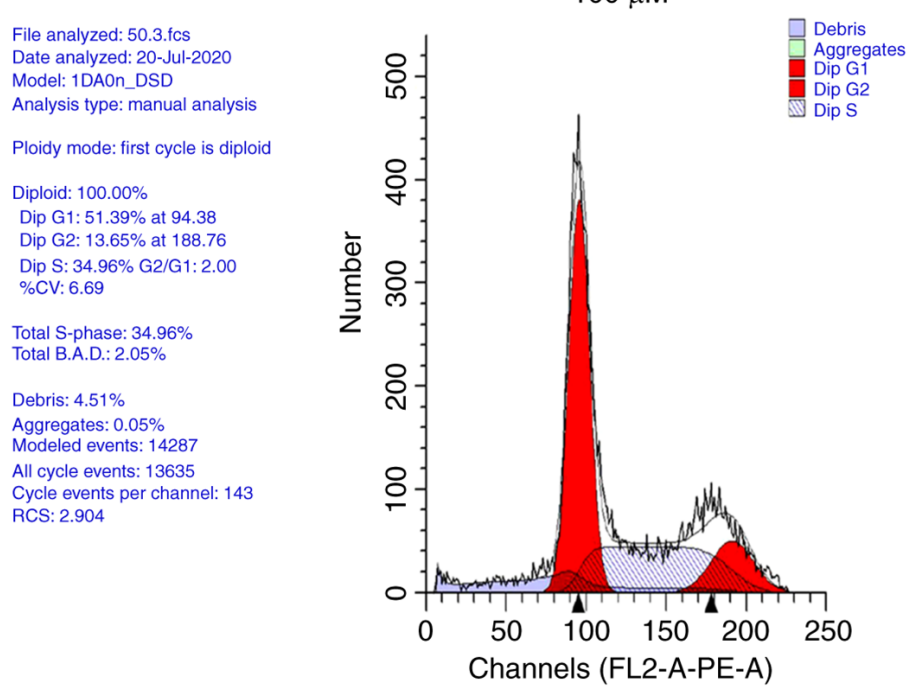

File analyzed: $50.3 . \mathrm{fcs}$ Date analyzed. 20-Jul-2020 Analysis type: manual analysis

Ploidy mode: first cycle is diploid

Diploid: $100.00 \%$ Dip S: $34.96 \%$ G2/G1: 2.00

Channels (FL2-A-PE-A)

File analyzed: 100-3.fos Date analyzed: 20-Jul-2020 Model: 1DAOn_DSD Analysis type: manual analysis Ploidy mode: first cycle is diploid Diploid: $100.00 \%$ Dip G1: $51.65 \%$ at 95.54 Dip G2: $13.61 \%$ at 191.07 Dip S: $34.74 \%$ G2/G1: 2.00 $\%$ CV: 6.85

Total S-phase: $34.74 \%$ Total B.A.D.: $5.37 \%$

Debris: $11.97 \%$ Aggregates: $0.00 \%$ Modeled events: 13774 All cycle events: 12126 Cycle events per channel: 126 RCS: 2.444
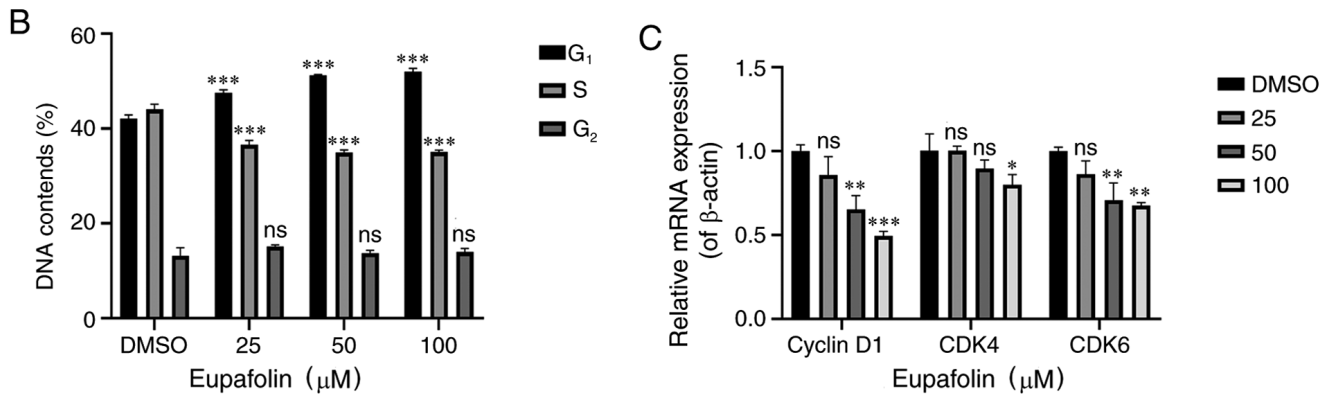

Figure 4. Eupafolin induces $\mathrm{G}_{0} / \mathrm{G}_{1}$ phase arrest in EO771 cells. (A) Flow chart showing the distribution of the proportions of different cell cycles. (B) Eupafolin treatment caused cell cycle arrest of EO771 cells at the $\mathrm{G}_{0} / \mathrm{G}_{1}$ phase. (C) Extracted RNA was used to determine the mRNA levels of cell cycle-associated genes, cyclin D1, CDK4 and CDK6. Data represent the results of three experiments and are expressed as the mean $\pm \mathrm{SD} . \mathrm{ns}, \mathrm{P}>0.05 ;{ }^{* * *} \mathrm{P}<0.001 ;{ }^{* *} \mathrm{P}<0.01 ;{ }^{*} \mathrm{P}<0.05$.

The anti-breast cancer proliferation activity of Eupafolin is shown in Fig. 1. Within $48 \mathrm{~h}$ of treatment, the inhibitory effect of Eupafolin increased in a time- and dose-dependent manner. MMP degrades various protein components in the extracellular matrix, destroys the histological barrier of tumor cell invasion, and plays a key role in tumor invasion and metastasis (15). VEGF is abnormally expressed in liver cancer and plays an important role in liver cancer neovascularization and tumor growth (5). The treatment of cancer by targeting VEGF and its receptor, VEGFR, is a much-explored topic in drug research (16). After treatment with different concentrations of Eupafolin, the migration and invasion ability of breast cancer cells decreased significantly. Furthermore, the mRNA levels of associated genes were detected using RT-qPCR, and the present data showed that mRNA levels of MMP2, MMP9 and VEGFA were significantly decreased (Fig. 2). Caspase 3 is the most important terminal splicing enzyme in apoptosis and plays an important part in the cell-killing mechanism (17). In addition, Bcl-2 was one of the first members of the $\mathrm{Bcl}-2$ protein family reported to regulate cell death. In cancer, Bcl-2 
A
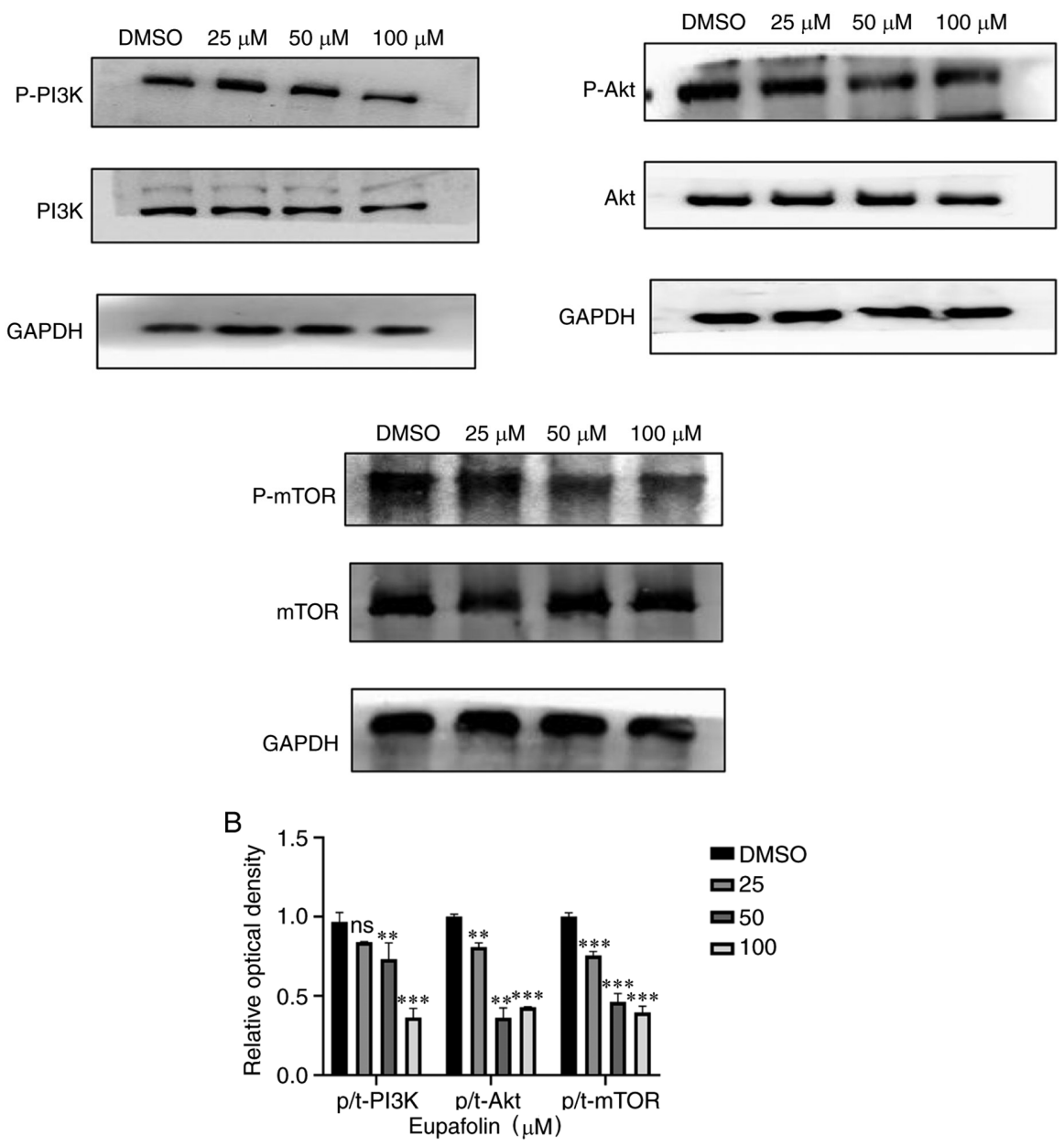

Figure 5. Eupafolin regulates the PI3K/Akt/mTOR pathway in EO771 cells. (A) EO771 cells were treated with 25, 50 and $100 \mu \mathrm{M}$ Eupafolin for $24 \mathrm{~h}$; the proteins were extracted and the associated protein phosphorylation (p)-PI3K, PI3K, p-Akt, Akt, mTOR and p-mTOR were detected. (B) Western blots were analyzed using ImageJ software. Comparison with protein bands normalized to GAPDH. Data are expressed as the mean $\pm \mathrm{SD}$. $\mathrm{ns}, \mathrm{P}>0.05 ;{ }^{* * * *} \mathrm{P}<0.001 ;$ ${ }^{* * *} \mathrm{P}<0.01$

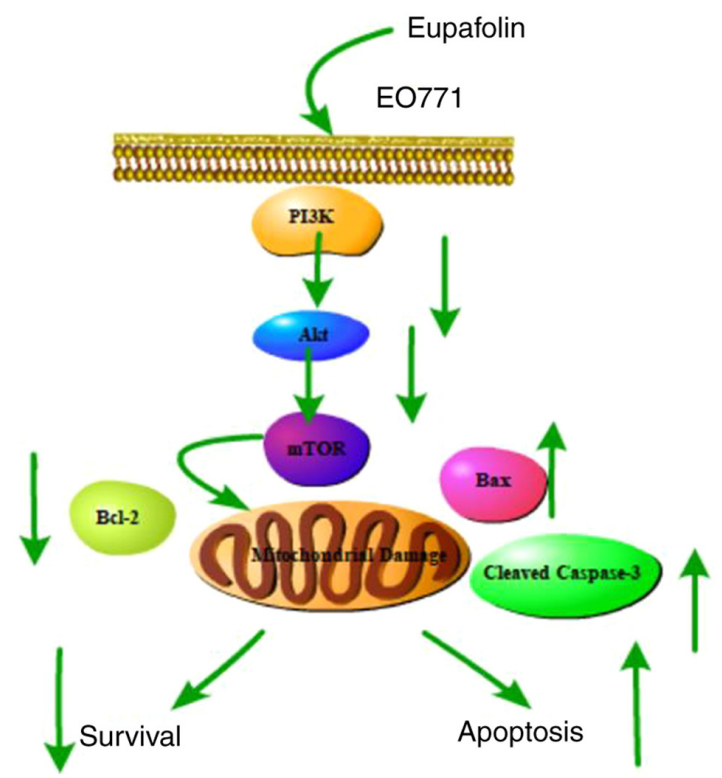

Figure 6. Schematic diagram illustrating that Eupafolin treatment induces downregulation of Bcl-2, and up-regulation of Bax and cleaved caspase 3 in EO771 cells. Eupafolin changes PI3K signal transduction by dephosphorylating PI3K, Akt, and mTOR phosphorylation products, thereby leading to PI3K/Akt/mTOR downregulation, and the regulation of cell survival and apoptosis. can prevent apoptosis of some cells and is highly expressed in cancer cells that occur in lymph nodes and other organs of the immune system (18). The balance between Bcl-2 and Bax protein determines cell survival or apoptosis. Flow cytometry results in the present study revealed that the apoptosis rate of cells increased significantly. Moreover, protein levels of the pro-apoptotic proteins, Bax and cleaved caspase 3, increased significantly, whereas protein levels of Bcl-2 decreased significantly after Eupafolin treatment (Fig. 3). The decrease or resistance of cell apoptosis often leads to further malignancy (19). Therefore, in some studies, it was shown that the apoptosis rate of tumor cells can be increased by activating or inhibiting various signaling pathways $(20,21)$. Naringin has been shown to inhibit the PI3K/Akt/mTOR pathway and promote the apoptosis of thyroid cancer cells (22), whereas hyperoside-induced breast cancer cell apoptosis is achieved via the reactive oxygen species (ROS)-mediated NF- $\mathrm{kB}$ signaling pathway (23).

Several studies have shown that there are critically important phases in the cell cycle: $G_{1}$ to $S$ and $G_{2}$ to $M$, which occur in a period of complex and active molecular level changes, which are easily affected by external conditions. If tumor growth needs to be suitably controlled, it can be achieved via two mechanisms. Cyclin-dependent protein 
kinases are a group of serine/threonine protein kinases, which can drive the cell cycle. Each cyclin-dependent kinase (CDK) binds to a different type of cyclin to form a complex, which regulates the transition of cells from the $G_{1}$ to the $S$ phase, or from the $G_{2}$ to the $M$ phase and exit from the $M$ phase. Increased expression of CDK and cyclin has been observed in most cancer cells; the increased activity of CDK may be associated with uncontrolled cell proliferation (24). When cells are damaged, the CDK inhibitor genes are upregulated, and the $\mathrm{G}_{1}$ phase-associated CDK complex is downregulated. Among them, both CDK4 and CDK6 can bind to cyclin D1 and play an important role in the $\mathrm{G}_{1} / \mathrm{S}$ phase (25). If the expression of $G_{1}$ cell cycle-associated proteins increases significantly, it causes the abnormal proliferation of tumor cells (26). Because the expression of CDK inhibitors in these cells may be insufficient, the $\mathrm{G}_{1}$ phase of the cell cycle arrest provides cells with a repair mechanism or follows the pathological changes (27). Therefore, targeting the cell-cycle disorders have been considered promising targets for cancer treatment (28). Results from flow cytometry showed that cells in the $\mathrm{G}_{0} / \mathrm{G}_{1}$ phase increased and those in the $S$ phase decreased in Eupafolin-treated cells. To the best of our knowledge, cancer occurs or develops through the activation of CDKs that regulate the cell cycle. Inhibiting the expression or function of CDK is becoming one of the promising strategies in cancer treatment (24). Among these proteins, cyclin D1, CDK4 and CDK6 are the key regulators of the $G_{0}-G_{1}$ phase (29). It was found that the mRNA levels of CDK4, CKD6 and cyclin D1 were decreased when cells were treated with $100 \mu \mathrm{M}$ Eupafolin, thus preventing the progression of the cell cycle from the $G_{1}$ to $S$ phase (Fig. 4).

To further understand the underlying anti-cancer mechanisms of Eupafolin in breast cancer cells, western blotting was performed to determine the proteins of the $\mathrm{PI} 3 \mathrm{~K} / \mathrm{Akt} / \mathrm{mTOR}$ pathway that were involved in tumor cell growth, differentiation, and apoptosis $(22,30)$. Generally, the expression of Akt in tumors is higher when compared with that in normal tissue, and the activation of Akt can activate many downstream target genes and regulate cell proliferation and cycle through several signaling pathways (31). Many studies have shown that the PI3k/Akt/mTOR pathway, as a therapeutic target, can effectively inhibit the further progression of tumors, including breast cancer, non-small cell lung cancer, esophageal cancer, gastric cancer and liver cancer (32). Astragaloside IV upregulates Nrf2 through the $\mathrm{PI} 3 \mathrm{~K} / \mathrm{Akt} / \mathrm{mTOR}$ signaling pathway to regulate inflammation and oxidative stress, thereby effectively inhibiting breast cancer cell metastasis (12). The combined use of compound Sophora flavescens and gefitinib can upregulate autophagy in lung cancer by inhibiting the PI3K/Akt/mTOR pathway (33). In addition, in animal models, the addition of Akt allosteric inhibitors and dual PI3K and mTOR inhibitors can inhibit the PI3K/Akt/mTOR pathway and inhibit the growth of esophageal cancer (34). These findings are consistent with the present results. In the present study, results from western blotting indicated that Eupafolin decreased the phosphorylation of PI3K, Akt, and mTOR proteins, but did not change their total protein levels (Fig. 5).

Taken together, the present findings showed that Eupafolin significantly inhibited the proliferation and migration of breast cancer cells, promoted apoptosis, and caused $\mathrm{G}_{0} / \mathrm{G}_{1}$ phase arrest, which was achieved by inhibiting the PI3K/Akt/mTOR pathway (Fig. 6). Thus, these results provide a theoretical basis for the use of Eupafolin in subsequent clinical trials. However, further research is needed to identify whether Eupafolin inhibits the growth of breast cancer cells through other pathways and target genes.

\section{Acknowledgements}

Not applicable.

\section{Funding}

This study was supported by Jilin Province Science and Technology Development Project (grant no. 20200703014ZP).

\section{Availability of data and materials}

The datasets used and/or analyzed during the current study are available from the corresponding author on reasonable request

\section{Authors' contributions}

JW, XZ, JZ and YD conceptualized the study and performed experimental research. XZ, SH and HP generated and analyzed the data. JW, XZ, BY and QL performed data analysis and edited the manuscript. All authors read and approved the final manuscript.

\section{Ethics approval and consent to participate}

Not applicable.

\section{Patient consent for publication}

Not applicable.

\section{Competing interests}

The authors declare that they have no competing interests.

\section{References}

1. Watkins EJ: Overview of breast cancer. JAAPA 32: 13-17, 2019.

2. Gao W, Ge S and Sun J: Ailanthone exerts anticancer effect by up-regulating miR-148a expression in MDA-MB-231 breast cancer cells and inhibiting proliferation, migration and invasion. Biomed Pharmacother 109: 1062-1069, 2019.

3. Chung KS, Choi JH, Back NI, Choi MS, Kang EK, Chung HG, Jeong TS and Lee KT: Eupafolin, a flavonoid isolated from Artemisia princeps, induced apoptosis in human cervical adenocarcinoma HeLa cells. Mol Nutr Food Res 54: 1318-1328, 2010.

4. Jiang H, Wu D, Xu D, Yu H, Zhao Z, Ma D and Jin J: Eupafolin exhibits potent anti-angiogenic and antitumor activity in hepatocellular carcinoma. Int J Biol Sci 13: 701-711, 2017.

5. Fan X, Tao J, Cai Z, Fredimoses M, Wu J, Jiang Z, Zhang K and Li S: Eupafolin suppresses esophagus cancer growth by targeting T-LAK cell-originated protein kinase. Front Pharmacol 10: 1248, 2019.

6. Liu K, Park C, Chen H, Hwang J, Thimmegowda NR, Bae EY, Lee KW, Kim HG, Liu H, Soung NK, et al: Eupafolin suppresses prostate cancer by targeting phosphatidylinositol 3-kinase-mediated Akt signaling. Mol Carcinog 54: 751-760, 2015. 
7. Am JU, Gong WJ, Su Y and Mou ZB: Imperatorin shows selective antitumor effects in SGC-7901 human gastric adenocarcinoma cells by inducing apoptosis, cell cycle arrest and targeting $\mathrm{PI} 3 \mathrm{~K} / \mathrm{Akt} / \mathrm{m}$-TOR signalling pathway. J BUON 22: 1471-1476, 2017.

8. Zhang Y, Zhang R and Ni H: Eriodictyol exerts potent anticancer activity against A549 human lung cancer cell line by inducing mitochondrial-mediated apoptosis, G2/M cell cycle arrest and inhibition of m-TOR/PI3K/Akt signalling pathway. Arch Med Sci 16: 446-452, 2019

9. Zhao JG, Zhang L, Xiang XJ, Yu F, Ye WL, Wu DP, Wang JF and Xiong JP: Amarogentin secoiridoid inhibits in vivo cancer cell growth in xenograft mice model and induces apoptosis in human gastric cancer cells (SNU-16) through G2/M cell cycle arrest and PI3K/Akt signalling pathway. J BUON 21: 609-617, 2016.

10. Livak KJ and Schmittgen TD: Analysis of relative gene expression data using real-time quantitative PCR and the 2(-Delta Delta C(T)) method. Methods 25: 402-408, 2001.

11. Thorat MA and Balasubramanian R: Breast cancer prevention in high-risk women. Best Pract Res Clin Obstet Gynaecol 65: 18-31, 2020.

12. Zhang XQ, Yao C, Bian WH, Chen X, Xue JX, Zhu ZY, Ying Y, $\mathrm{Xu}$ YL and Wang C: Effects of Astragaloside IV on treatment of breast cancer cells execute possibly through regulation of Nrf2 via PI3K/AKT/mTOR signaling pathway. Food Sci Nutr 7 : 3403-3413, 2019

13. Lai ZR, Ho YL, Huang SC, Huang TH, Lai SC, Tsai JC, Wang CY, Huang GJ and Chang YS: Antioxidant, anti-inflammatory and antiproliferative activities of Kalanchoe gracilis (L.) DC Stem. Am J Chin Med 39: 1275-1290, 2011.

14. Han MA, Min KJ, Woo SM, Seo BR and Kwon TK: Eupafolin enhances TRAIL-mediated apoptosis through cathepsin S-induced down-regulation of Mcl-1 expression and AMPK-mediated Bim up-regulation in renal carcinoma Caki cells. Oncotarget 7: 65707-65720, 2016.

15. Wang X, Hu Z, Wang Z, Cui Y and Cui X: Angiopoietin-like protein 2 is an important facilitator of tumor proliferation, metastasis, angiogenesis and glycolysis in osteosarcoma. Am J Trans Res 11: 6341-6355, 2019.

16. Aksenenko MB, Palkina NV, Sergeeva ON, Yu Sergeeva E, Kiriehenko AK and Ruksha TG: miR-155 overexpression is followed by downregulation of its target gene, NFE2L2, and altered pattern of VEGFA expression in the liver of melanoma B16-bearing mice at the premetastatic stage. Int J Exp Pathol 100: 311-319, 2019.

17. Shalini S, Dorstyn L, Dawar S and Kumar S: Old, new and emerging functions of caspases. Cell Death Differ 22: 526-539, 2015.

18. Zeng C, Ke Z, Song Y, Yao Y, Hu X, Zhang M, Li H and Yin J: Annexin A3 is associated with a poor prognosis in breast cancer and participates in the modulation of apoptosis in vitro by affecting the Bcl-2/Bax balance. Exp Mol Pathol 95: 23-31, 2013

19. Cao Z, Zhang G, Xie C and Zhou Y: miR-34b regulates cervical cancer cell proliferation and apoptosis. Artif Cells Nanomed Biotechnol 47: 2042-2047, 2019.

20. Liu R, Chen Y, Shou T, Hu J, Chen J and Qing C: TRIM67 promotes NFKB pathway and cell apoptosis in GA-13315-treated lung cancer cells. Mol Med Rep 20: 2936-2944, 2019.
21. Wu G, Zheng H, Xu J, Guo Y, Zheng G, Ma C, Hao S, Liu X, Chen H, Wei S, et al: miR-429 suppresses cell growth and induces apoptosis of human thyroid cancer cell by targeting ZEB1. Artif Cells Nanomed Biotechnol 47: 548-554, 2019.

22. Zhou J, Xia L and Zhang Y: Naringin inhibits thyroid cancer cell proliferation and induces cell apoptosis through repressing PI3K/AKT pathway. Pathol Res Pract 215: 152707, 2019.

23. Qiu J, Zhang T, Zhu X, Yang C, Wang Y, Zhou N, Ju B, Zhou T, Deng G and Qiu C: Hyperoside induces breast cancer cells apoptosis via ROS-Mediated NF- $\kappa \mathrm{B}$ signaling pathway. Int J Mol Sci 21: 131, 2019.

24. Noori S and Hassan ZM: Tehranolide inhibits proliferation of MCF-7 human breast cancer cells by inducing G0/G1 arrest and apoptosis. Free Radic Biol Med 52: 1987-1999, 2012.

25. Suryavanshi S, Choudhari A, Raina P and Kaul-Ghanekar R: A polyherbal formulation, $\mathrm{HC} 9$ regulated cell growth and expression of cell cycle and chromatin modulatory proteins in breast cancer cell lines. J Ethnopharmacol 242: 112022, 2019.

26. Ahn H, Im E, Lee DY, Lee HJ, Jung JH and Kim SH: Antitumor Effect of pyrogallol via miR-134 mediated $S$ phase arrest and inhibition of PI3K/AKT/Skp2/cMyc signaling in hepatocellular carcinoma. Int J Mol Sci 20: 3985, 2019.

27. Hunter T and Pines J: Cyclins and cancer. II: Cyclin D and CDK inhibitors come of age. Cell 79: 573-582, 1994

28. Park W, Park S, Song G and Lim W: Inhibitory effects of osthole on human breast cancer cell progression via induction of cell cycle arrest, mitochondrial dysfunction, and ER stress. Nutrients 11: 2777, 2019.

29. Gao X, Wang Y, Li Y, Wang Y, Yan M, Sun H, Chen S and Pan X: Huganpian, a traditional Chinese medicine, inhibits liver cancer growth in vitro and in vivo by inducing autophagy and cell cycle arrest. Biomed Pharmacother 120: 109469, 2019.

30. Liu X, Sun L, Zhang S, Zhang S and Li W: GINS2 facilitates epithelial-to-mesenchymal transition in non-small-cell lung cancer through modulating PI3K/Akt and MEK/ERK signaling. J Cell Physiol 235: 7747-7756, 2020.

31. Guo L and Yang T: Oxymatrine inhibits the proliferation and invasion of breast cancer cells via the PI3K pathway. Cancer Manag Res 11: 10499-10508, 2019.

32. Murugan AK: Special issue: PI3K/Akt signaling in human cancer. Semin Cancer Biol 59: 1-2, 2019.

33. Zhang J, Qu Z, Yao H, Sun L, Harata-Lee Y, Cui J, Aung TN, Liu X, You R, Wang W, et al: An effective drug sensitizing agent increases gefitinib treatment by down regulating PI3K/Akt/mTOR pathway and up regulating autophagy in non-small cell lung cancer. Biomed Pharmacother 118: 109169, 2019.

34. Shi N, Yu H and Chen T: Inhibition of esophageal cancer growth through the suppression of PI3K/AKT/mTOR signaling pathway. Onco Targets Ther 12: 7637-7647, 2019.

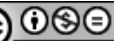

This work is licensed under a Creative Commons Attribution-NonCommercial-NoDerivatives 4.0 International (CC BY-NC-ND 4.0) License. 